\title{
Personalised Medicine in Modern Era
}

\section{Abolfazl Movafagh}

Asian Pac J Cancer Biol, 1 (2), 31-32

\section{Dear Editor}

Personalised Medicine is a medical procedure that separates patients into different groups-with medical decisions, practices, interventions and/or products being tailored to the individual patient based on their predicted response or risk of disease. The terms personalized medicine, precision medicine, stratified medicine are used interchangeably to describe this concept.

the term has risen in usage in recent years given the growth of new diagnostic and informatics approaches that provide understanding of the molecular basis of disease, particularly genomics. This provides a clear evidence base on which to stratify (group) related patients. Every person has a unique variation of the human genome. Although most of the variation between individuals has no effect on health, an individual's health stems from genetic variation with behaviors and influences from the environment.

Modern advances in personalized medicine rely on technology that confirms a patient's fundamental biology, DNA, RNA, or protein, which ultimately leads to confirming disease. For example, personalised techniques such as genome sequencing can reveal mutations in DNA that influence diseases ranging from cystic fibrosis to cancer. Another method, called RNA-seq, can show which RNA molecules are involved with specific diseases. Unlike DNA, levels of RNA can change in response to the environment. Therefore, sequencing RNA can provide a broader understanding of a person's state of health. Recent studies have linked genetic differences between individuals to RNA expression, translation, and protein levels.

The concepts of personalised medicine can be applied to new and transformative approaches to health care. Personalised health care is based on the dynamics of systems biology and uses predictive tools to evaluate health risks and to design personalised health plans to help patients mitigate risks, prevent disease and to treat it with precision when it occurs. The concepts of personalised health care are receiving increasing acceptance with the Veterans Administration committing to personalised, proactive patient driven care for all veterans.
Submission Date: 03/15/2016 Acceptance Date: 05/25/2016

In order for physicians to know if a mutation is connected to a certain disease, researchers often do a study called a "genome-wide association study" (GWAS). A GWAS study will look at one disease, and then sequence the genome of many patients with that particular disease to look for shared mutations in the genome. Mutations that are determined to be related to a disease by a GWAS study can then be used to diagnose that disease in future patients, by looking at their genome sequence to find that same mutation. The first GWAS, conducted in 2005, studied patients with age-related macular degeneration (ARMD). It found two different mutations, each containing only a variation in only one nucleotide (called single nucleotide polymorphisms, or SNPs), which were associated with ARMD. GWAS studies like this have been very successful in identifying common genetic variations associated with diseases. As of early 2014, over 1,300 GWAS studies have been completed.

\section{References}

1. Stratified, personalised or P4 medicine: a new direction for placing the patient at the centre of healthcare and health education (Technical report). Academy of Medical Sciences. May 2015. Retrieved 6 Jan 2016.

2. Jump up to "Many names for one concept or many concepts in one name?". PHG Foundation. Retrieved 6 Jan 2015.

3. Jump up Egnew, Thomas (1 Mar 2009). "Suffering, Meaning, and Healing: Challenges of Contemporary Medicine". Annals of Family Medicine. 7 (2): 170-175. doi:10.1370/ afm.943. Retrieved 6 January 2016.

4. Jump up "The Case for Personalized Medicine" (PDF). Personalized Medicine Coalition. 2014. Retrieved 6 Jan 2016.

5. Jump up Smith, Richard (15 Oct 2012). "Stratified, personalised, or precision medicine". British Medical Journal. Retrieved 6 January 2016.

6. Jump up to: "Personalized Medicine 101". Personalized Medicine Coalition. Retrieved 26 April 2014.

7. Jump up to: Dudley, J; Karczewski, K. (2014). Exploring Personal Genomics. Oxford: Oxford University Press.

8. Jump up to: "Personalized Medicine 101: The Science". Personalized Medicine Coalition. Retrieved 26 April 2014.

9. Jump up Lu, YF; Goldstein, DB; Angrist, M; Cavalleri, G

Corresponding Author:

Dr. Abolfazl Movafagh

Department of Medical Genetics, Cancer Research Center, Shoha Hospital, School of Medicine, Shahid Beheshti University of Medical

Science, Tehran, Iran.

Email: movafagh.a@sbmu.ac.ir 
(24 July 2014). "Personalized medicine and human genetic diversity". Cold Spring Harbor perspectives in medicine. 4 (9): a008581. doi:10.1101/cshperspect.a008581. PMID 25059740 .

10. Jump up Alexis Battle, Sara Mostafavi, Xiaowei Zhu, James B. Potash, Myrna M. (2014). "Characterizing the genetic basis of transcriptome diversity through RNA-sequencing of 922 individuals". Genome research. 24 (1): 14-24. doi:10.1101/gr.155192.113. PMID 24092820.

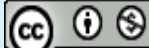

This work is licensed under a Creative Commons AttributionNon Commercial 4.0 International License. 